\title{
Mecanismos de control externo en las universidades de Chile: una mirada a partir del enfoque contractual
}

\author{
Ganga Contreras, Francisco* \\ Burotto, Juan Felix**
}

\section{Resumen}

El propósito primordial que anima este trabajo, está asociado a la aplicación del enfoque contractual o de agencia en las universidades de Chile, de uno de sus mecanismos más importantes, como lo es el control externo. Para lograr obtener la percepción de agente y principal de estas instituciones, se utiliza una población de 54 casas de estudios, con una muestra que alcanza el $54 \%$. La confianza utilizada fue del $95 \%$ y el error muestral de 8,11 ; tomando para ello varianza máxima. Entre los principales resultados y conclusiones, se pueden citar el hecho de que los diferentes actores no le dan mucha relevancia a los mecanismos de control externo, pero además dejan de manifiesto que éstos son ineficientes, siendo muy mal evaluados los organismos públicos que deben ejercer algún nivel de supervisión y alineamiento en las universidades. De esta forma, se podría colegir que los sistemas de control interno deben tomar mayor fuerza, de modo tal, que se pueda compensar la mínima gravitación que tienen los mecanismos de control externo.

Palabras clave: Mecanismos alineadores, sistemas de control, universidades, teoría de agencia, agente y principal.

Recibido: 15-03-10. Aceptado: 01-09-10

* Administrador Público y los grados de:Licenciado en Administración, Magíster en Administración de Empresas, DEA en Organización de Empresas y Doctor en Administración de Empresas. Actualmente es académico jornada completa de la Universidad de Los Lagos-Chile. Correo postal: Chinquihue Km 6, Universidad de Los Lagos, Puerto Montt, Chile.

E-mail: fganga@ulagos.cl

** Abogado y Licenciado en Ciencias Jurídicas y Sociales. Epistemólogo y Miembro de Honor del Instituto Literario y Cultural Hispánico, Universidad del Estado de California, California, USA. Actualmente es académico jornada completa de la Universidad de Los Lagos-Chile. Correo postal: Chinquihue Km 6, Universidad de Los Lagos, Puerto Montt, Chile.

E-mail: jburotto@ulagos.cl 
Mecanismos de control externo en las universidades de Chile...

Ganga Contreras, Francisco y Burotto, Juan Felix

\title{
External Control Mechanisms in Chilean Universities: A Perspective Based on the Contractual Approach
}

\begin{abstract}
The primary purpose underlying this work is associated with implementing the contractual or agency approach at Chilean universities for one of their most important mechanisms: external control. In order to obtain the perceptions of agent and principal at these institutions, a population of 54 houses of study was used with a sample that reached $54 \%$. Confidence was $95 \%$ with a sampling error of 8.11 , taking maximum variance for it. Among the main findings and conclusions, it can be mentioned that the different actors do not give much importance to external control mechanisms, but also, they make it clear that these mechanism are inefficient, and the public bodies that should exercise some degree of supervision and alignment in the universities receive a very poor evaluation. Thus, it could be deduced that internal control systems should become stronger to compensate for the minimum weight of the external control mechanisms.
\end{abstract}

Key words: Alignment mechanisms, control systems, universities, agency theory, agent and principal.

\section{Introducción}

Dentro del estudio de los gobiernos corporativos y sus articulaciones internas, hay un creciente interés porque existan sustratos teóricos, epistemológicamente sustentables, que permitan investigaciones que sobrepasen la mera descripción de dichas organizaciones y que, por lo mismo, tengan capacidad de formar parte de un intertexto científico que, como bien se sabe, no termina de asentarse en el campo de las denominadas Ciencias Sociales, Ciencias Humanas o, también, Ciencias de la Conducta.

En el presente trabajo y conteste con lo recién enunciado, convergen dos intereses. El primero es hacer aplicable el modelo propuesto por la Teoría de Agencia o Contractual, en uno de sus rubros más dinámicos, el del control $\mathrm{y}$, particularmente, el del control externo. Como es de dominio general, la Teoría de Agencia entiende que cualesquiera emprendimientos en el área de las corporaciones y empresas y desde cierto grado de complejidad en adelante, es obligatorio instalar un modelo que acoja la delegación imprescindible entre un Principal, verbigracia, la Junta de accionistas, los dueños, etc. y un Agente, verbigracia, un gerente. El supuesto que se esgrime, grosso modo, es que existe un potencial divorcio entre la propuesta del Principal y la conducta efectiva del Agente, pese a que éste había contratado coincidiendo con la propuesta del primero. Una de las fórmulas para contener o aminorar esta divergencia está en los mecanismos alineadores, internos y externos; en estos últimos estará el foco de nuestra investigación.

El campo a investigar lo constituye, en este empeño, el conjunto de las universidades chilenas, examinado por la vía de los gobiernos que se dan tales corporaciones. Por lo anterior, se buscó un diseño 
muestral que facilite la obtención de resultados, con un apropiado grado de confianza y un tolerable margen de error. Considerando los criterios anteriormente señalados, se utilizó para el análisis una población total de $\mathrm{N}=54$ universidades, sin embargo, la muestra quedó finalmente constituida por un total de 29 universidades (que corresponde al $54 \%$ del universo) de las cuales 11 son privadas y el resto tradicionales. La confianza utilizada fue del $95 \%$ y el error del $8,11 \%$, considerando para ello varianza máxima. Las características de las variables resultantes de la encuesta implicaron la utilización exclusivamente de pruebas no paramétricas para el análisis de los resultados.

Siguiendo a la Teoría, y luego de definir con precisión el universo-objeto de la investigación, existirá un Principal que, en la especie, lo representa cada Máximo Cuerpo Colegiado (MCC) - Junta Directiva, Directorio o Consejo Superior - y un Agente, que, sin excepción, el conjunto denomina Rector.

Como quedará de manifiesto, la complejidad de la cosa universitaria, arraigada en el seno de toda sociedad y, por cierto, en la chilena, sin perjuicio de su necesaria gravitación en dicha sociedad, hace que la investigación que se expone puede tener una vocación que la hace no sólo académica sino que, adicionalmente, la convierte en una herramienta más que indispensable a la hora de pensar la universidad. En este caso, la calidad y número de mecanismos de control externo ameritan un examen riguroso, que sea por la vía del financiamiento -concursalidad, negociación- o del control cualitativo - certificaciones periódicas de calidad, controles gubernamenta- les variopintos, protección al consumidor - existentes en Chile.

El testeo, que hace pie en percepciones, recibe un tratamiento estricto, con el plus que tales percepciones tienen el valor de ser emitidas por sujetos de alta calificación profesional y dentro del campo de quehaceres que, como se ha de suponer, resguardan como prioritario el mayor nivel de acercamiento a lo verdadero.

Como se expone en el corpus textual, se ratificará la existencia de los mecanismos externos; sin embargo, también queda precisado que la percepción de su gravitación de control por los actores de la Teoría es claramente menor a lo que podría ser un estándar esperable. Ello se analizará con mayor detenimiento en el trabajo y al momento de concluir.

\section{Concepto y mecanismos de control corporativo}

A juicio de Shleifer y Vishny (1995), el control corporativo es factible definirlo, strictu sensu, como el análisis y seguimiento de los medios por lo cuales los accionistas o "dueños" logran recibir una remuneración acorde a la inversión realizada en un proyecto determinado.

Para lograr las finalidades planteadas por los autores supracitados, esta función requiere evaluar los resultados de la organización y del director general, de forma tal, que se pueda asegurar un apropiado crecimiento institucional, protegiendo los intereses accionariales (Zahra y Pearce, 1989; Monks y Minow, 1995 y Forbes y Miliken, 1999).

Complementariamente Fogelberg (1980), estima que el control de una organización debe ser entendido como la ca- 
Mecanismos de control externo en las universidades de Chile...

Ganga Contreras, Francisco y Burotto, Juan Felix

pacidad de dirigir los asuntos organizacionales, afectando las decisiones políticas que se tomen, donde el control último viene predefinido por el prorrateo de acciones y la capacidad de cualquier "dueño" para influir en las decisiones que se tomen en el consejo de administración o máximo cuerpo colegiado.

Por lo tanto, el control corporativo está orientado a comparar las acciones planificadas por los inversionistas, al momento de decidir la materialización de determinadas inversiones y los resultados que se vayan produciendo o que se produzcan finalmente.

Desde la óptica de la Teoría de Agencia, el control corporativo posee herramientas que tienen como propósito alinear los comportamientos oportunistas del Agente que para el caso de las universidades en Chile es el rector, hacia los objetivos que tienen los máximos cuerpos colegiados (MCC) o Principal...

Dependiendo de donde se ejercen estos mecanismos, pueden ser clasificados en internos y externos. En el primer caso se encuentran principalmente las acciones que desarrollan los consejos de administración, la designación de comités de auditoria y los sistemas retributivos que se utilizan para compensar a los directivos; a su vez, respecto de los mecanismos de control externo, se distinguen los mercados de capitales (específicamente el mercado de control corporativo), el sistema legal, político y regulatorio y los mercados de factores y productos (Jensen, 1993; Fernández y Gómez-Ansón, 1998; Manjón, 2000; Fernández et al., 2001; Maroto y Melle, 2001; Cuervo et al., 2002; Maroto et al., 2006). Todos estos mecanismos conforman el denominado gobierno de la organización y tienen una serie de características que no son análogas entre los países, dado que dependen del cuerpo normativo de cada uno de ellos, así como también de las diversas culturas empresariales.

Como esta indagación se concentra en los mecanismos de control externo, a continuación se revisarán sus principales características.

\section{Mecanismos de control externo (MCE)}

Los MCE juegan un rol relevante en el enfilamiento de intereses, entre los dueños y los directivos, especialmente cuando los mecanismos de control interno son ineficientes, inadecuados o fallan en su aplicación (Hoskisson y Turk, 1990). Por lo tanto, el objetivo de los MCE está orientado a restringir la capacidad de los gerentes para asumir y eventualmente mantener, comportamientos oportunistas. Estos MCE provienen del funcionamiento de los múltiples escenarios competitivos, que se encuentran inmersos en una economía de mercado.

Siguiendo a diversos autores, como Jensen y Mecling (1976), Fama (1980), Demsetz (1983), Hart (1983), Jensen (1993) y Shivdasani (1993), Azofra y Santamaría (2004), es posible identificar los principales MCE, entre los cuales se pueden citar: el mercado de capital humano, el mercado de donaciones, el mercado de capitales, el mercado de productos y servicios, y el marco legal, político y reglamentario. Una sucinta explicación se presenta a continuación.

a) El mercado de capital humano: en el cual los directivos son contratados y cuya 
retribución (valor recibido por el directivo) dependerá del prestigio que haya logrado alcanzar. Este mercado funciona como mecanismo de supervisión, que es subproducto de los esfuerzos mancomunados de propietarios y directivos, por tratar de alcanzar al máximo su correspondiente satisfacción (Ocaña y Salas, 1983; Lozano, 2005).

b) El mercado de donaciones: este mecanismo es aplicable para el caso de las entidades no lucrativas, las que tendrán acceso a recursos financieros de parte de donantes, dependiendo de la mayor o menor eficiencia que muestren en su gestión y a la atractividad de su misión.

c) El mercado de capitales: corresponde a el lugar donde se establece el precio de las acciones de una empresa;

d) El mercado de productos y servicios: instancia donde se evalúa la administración de los recursos por parte de la empresa, pues, el consumidor ejercerá su "soberanía", eligiendo aquellas empresas que considere más eficientes y;

e) El marco legal, político y reglamentario: en este se pueden regular, precisar y estabilizar ciertos aspectos del comportamiento de los directivos.

\subsection{Mecanismos de control externo en las universidades}

Entre los mecanismos de control externo podemos discutir sobre aquellos relacionados con los sistemas públicos de control y algunos aspectos relacionados con estos.

\section{a) Sistema de financiamiento}

Existen múltiples mecanismos que utilizan los países para permitir el financiamiento de sus universidades; pero en términos globales es factible analizarlo desde tres puntos de vistas:

1. Asignación de fondos vía resultados: en este caso, los fondos se entregan en consideración al desempeño experimentado por las respectivas casas de estudios. Para ello se utilizan una serie de indicadores que miden aspectos vinculados con la tres funciones tradicionales que se le reconocen a las universidades, vale decir, docencia (formación de graduados y postgraduados), investigación y extensión (Salmi y Alcalá, 1998).

2. Asignación de fondos vía costes: en esta situación se utilizan fórmulas que posibilitan la medición de la cantidad de alumnos, respecto de parámetros asociados a los costes incurridos en la formación académica, tales como, características de los alumnos, número de facultades, departamentos y/o programas, cantidad y calidad de académicos y docentes, metros cuadrados construidos, inversiones en tecnología; entre otros.

3. Asignación de fondos vía negociación: esta modalidad implica la asignación de fondos sobre la base de un proceso de negociación, que contempla como principal parámetro distributivo, los recursos que históricamente ha recibido una institución.

Hace ya algunos años, los investigadores Albrecht y Ziderman (1992) descubrieron en una muestra de treinta y seis países seleccionados, que el principal mecanismos de asignación de recursos era aquel que se realizaba vía negociación y estaba constituido en su mayoría por países en vía de desarrollo; un porcentaje menor lo hacía vía costos, listado que 
Mecanismos de control externo en las universidades de Chile...

Ganga Contreras, Francisco y Burotto, Juan Felix

coincidía con los países industrializados; y muy pocos vía resultados (Diagrama 1).

Chile tiene un sistema de financiamiento bastante peculiar, el cual se ha mantenido a través de los años. Este mecanismo de asignación de recursos, ha generado un amplio debate, que ha incorporado fuertemente el tema del rol que debe cumplir el Estado en materia de financiamiento. En esta lógica, en las últimas dos décadas se han confrontado dos perspectivas: una que se inspira en las concepciones neoliberales y que postula, por lo mismo, el autofinanciamiento de las casas de estudios superiores, pues, se asume que estas organizaciones ofertan un servicio que finalmente se transforma en un bien de uso personal, y por lo tanto, debe ser pagado por quienes usufructúan de este beneficio. Sin embargo, también están aquellos que opinan que la universidad es un ente público que no solo beneficia a quienes reciben el servicio educacional, sino que ellas desarroIlan acciones que se constituyen en motor de desarrollo de toda una comunidad; en este sentido, es imperativo que el Estado sea capaz de asumir un rol protagónico en el financiamiento de la función universitaria, no dejando esta estratégica labor en manos del mercado.

A pesar de todas las discusiones, se sigue imponiendo el modelo basado principalmente en la asignación de recursos, vía funcionamiento del mercado (captación de alumnos), prueba de ello, es que no existe ninguna universidad tradicional que sea financiada por el Estado, más allá del $30 \%$ de su presupuesto. En este orden de cosas, los recursos financieros que proporciona el Estado, según el DFL de educación $\mathrm{N}^{\circ} 4$ de 1981 , se entregan a las universidades a través de dos vías: por un lado está el llamado

\section{Diagrama 1 \\ Mecanismos de asignación de recursos en treinta y seis países seleccionados}

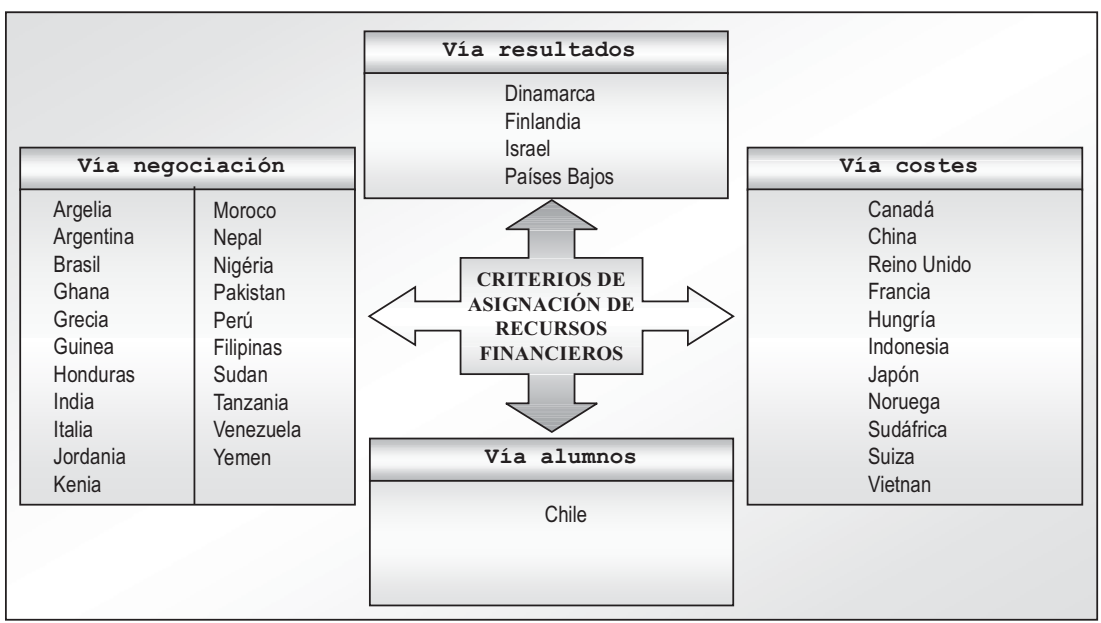

Fuente: Diseño propio, basado en Albrecht y Ziderman (1992). 
aporte fiscal directo (AFD), fondo al cual tienen acceso solamente las veinticinco universidades que pertenecen al Consejo de Rectores de Universidades Chilenas ${ }^{1}$. Por otra parte, está el aporte fiscal indirecto (AFI), al que pueden acceder la totalidad de las casas de estudios que participan en el sistema universitario, siempre y cuando, logren matricular alumnos que estén en el grupo de los 27.000 mejores puntajes logrados en la prueba de selección universitaria (PSU).

Junto a lo anterior, está el denominado crédito fiscal (actualmente crédito universitario), el que sigue la premisa de que las universidades deben cobrar aranceles que reflejen el costo real de la docencia y el beneficio privado que implica la formación profesional recibida por el alumno. Este mecanismo se creó para financiar a los estudiantes de escasos medios económicos, a través, de un sistema de préstamos con cargo a fondos de origen estatal.

Con posterioridad, se crearon nuevos mecanismos de financiamiento para la enseñanza superior, fundamentalmente, fondos concursables e incentivos tributarios para los aportes privados a la educación.

\section{b) Sistema de acreditación}

En el año 1992, se crea en Chile el Consejo Superior de Educación (CSE), por medio de la Ley 18.962, Orgánica Constitucional de Enseñanza (LOCE), con la finalidad de administrar, el nuevo sistema de supervisión de universidades e institutos profesionales, implicando por lo tanto, evaluar el grado de desarrollo de los proyectos institucionales, durante el período de acreditación ${ }^{2}$ que fija la respectiva Ley De acuerdo a el artículo 41 de la LOCE, este consejo tendrá las siguientes atribuciones:

- Pronunciarse sobre los proyectos institucionales que presenten las distintas universidades e institutos profesionales para los efectos de su reconocimiento oficial;

- Verificar progresivamente el desarrollo de los proyectos institucionales de conformidad a las normas de acreditación establecidas en esta ley;

- Establecer sistemas de examinación selectiva para las instituciones de educación sometidas a procesos de acreditación, salvo que el Consejo declare exentas determinadas carreras. Dicha exención no procederá respecto de aquellas carreras cuyos títulos profesionales requieren haber obtenido previamente a su otorgamiento el grado de licenciado.

- Esta examinación tendrá por objeto evaluar el cumplimiento de los planes y programas de estudio y el rendimiento de los alumnos;

- Recomendar al Ministro de Educación la aplicación de sanciones a las entidades en proceso de acreditación;

- Informar al Ministerio de Educación respecto de las materias establecidas en los artículos 18 y 19 de esta ley, en el plazo máximo de sesenta días contados desde la recepción de la solici-

1 Estas instituciones se conocen también como universidades tradicionales. 
Mecanismos de control externo en las universidades de Chile...

Ganga Contreras, Francisco y Burotto, Juan Felix

tud por parte del Ministerio. Si el Consejo no se pronunciare dentro de dicho plazo, se entenderá cumplido el trámite respectivo;

- Servir como órgano consultivo del Ministerio de Educación en las materias relacionadas con la presente ley;

- Designar al Secretario Ejecutivo el que permanecerá en el cargo mientras cuente con la confianza del Consejo;

- Designar comisiones ad-hoc en todos aquellos casos en que sea necesaria la asesoría de expertos en materias especiales o sobre aquellas en que por su trascendencia se encuentre involucrada la fe pública;

- Encomendar la ejecución de acciones o servicios a personas o instituciones públicas o privadas para el debido cumplimiento de sus funciones;

- Desarrollar toda otra actividad que diga relación con sus objetivos, y

- Establecer su reglamento interno de funcionamiento.

A medida que el CSE fue desarrollando sus labores, fueron muchas las entidades que se crearon y muchas las que fueron logrando su autonomía. Frente a esta realidad, surgió entonces, la necesidad de estructurar un sistema que permitiese evaluar la calidad, de las múltiples instituciones de educación superior autónomas; es así como en el año 1999, se crea la Comisión Nacional de Acreditación de Pregrado, cuyo función primordial está orienta- da a conducir los procesos experimentales de acreditación de carreras e instituciones y diseñar y proponer el sistema nacional de aseguramiento de la calidad de la Educación Superior. El propósito primordial del proceso de acreditación es asegurar la calidad, ofrecida tanto a nivel programático como institucional, por lo que debe ser entendido como un sistema permanente, aplicado en ciclos preestablecidos.

El tema de la calidad se encuentra en el centro de la agenda de prácticamente todos los sistemas de educación superior en el mundo. La calidad significa que sólo es posible su promoción y mejora con el concurso activo y el compromiso de los propios actores, es decir, una tarea compartida de las instituciones de educación superior.

Por ello, el trabajo de la CNAP tiene como foco prioritario el desarrollo de la capacidad institucional de autorregulación y la integración de académicos y profesionales en el diseño de criterios y procedimientos de evaluación. Las actividades que desarrolla la CNAP, se enmarcan en el Componente de Aseguramiento de la Calidad del Programa de Mejoramiento de la Calidad y la Equidad de la Educación Superior (MECESUP), que lleva a cabo el Ministerio de Educación con el apoyo del Banco Mundial. Por lo tanto, este organismo público, con el claro propósito de dar cumplimiento a sus obligaciones, asume tres ámbitos de acción:

2 Para no confundirlo con el proceso de acreditación voluntaria, impulsado a partir del año 1999 con la creación de la Comisión Nacional de Acreditación de Pregrado (CNAP), se le dio finalmente a este proceso, el nombre de licenciamiento. 
- Diseño y desarrollo de procesos experimentales de acreditación de carreras.

- Implementar procesos de acreditación institucional.

- Acciones de apoyo a la capacidad de autorregulación de las instituciones de educación superior.

La labor de la CNAP también incluye diversos estudios y recopilación de información de apoyo al desarrollo de las líneas de acción antes señaladas como la difusión y comunicación, orientada a dar a conocer los avances de la Comisión en materia de acreditación a las instituciones de educación superior (CNAP, 2006).

\section{Principales organismos públicos que ejercen control en las universidades de Chile}

Existen por lo menos cuatro organismos públicos que ejercen ciertos niveles de control en las diversas universidades de Chile. Estos son: Ministerio de Educación, Contraloría General de la República, Servicio Nacional del Consumidor e Inspección del Trabajo. NEDUC)

a) Ministerio de Educación (MI-

De acuerdo al propio Ministerio de Educación (2010), esta repartición pública tiene como misión: "asegurar un sistema educativo equitativo y de calidad que contribuye a la formación integral y permanente de las personas y al desarrollo del país, mediante la formulación e implementación de políticas, normas y regulación sectorial".

En cuanto a sus funciones, se distinguen las siguientes:
- Diseñar, normar, evaluar y supervisar la ejecución de las políticas, los planes y objetivos de desarrollo educacional y cultural, que permitan orientar el sistema educacional en todos sus niveles y modalidades y velar por su cumplimiento.

- Diseñar, proponer e implementar programas que contribuyan al desarrollo de las distintas áreas de conocimiento.

- Determinar, fijar, diseñar e implementar las políticas y programas de protección que se estimen necesarias y apropiadas para cautelar las garantizas constitucionales que consagran el derecho a la educación y a la libertad de enseñanza y velar por la aplicación estricta de la normativa con tal objeto.

- Asignar, distribuir y fiscalizar los recursos de las subvenciones estatales $y$, adicionalmente, focalizar otros recursos o elementos necesarios para el desarrollo de los distintos niveles y modalidades de educación, en conformación con la normativa vigente.

- Diseñar, implementar, fiscalizar y evaluar los programas de mejoramiento e innovaciones educativas, que apoyen las políticas ministeriales.

- Estimular el desarrollo educativo de los alumnos, focalizando los recursos con el objeto de apoyar significativamente a aquellos de mayor vulnerabilidad, de conformidad a la ley.

- Gestionar, asignar y monitorear las ayudas estudiantiles.

- Diseñar, desarrollar y adoptar las medidas para la aplicación del marco curricular y los planes y programas de estudio para los niveles prebásico, 
Mecanismos de control externo en las universidades de Chile...

Ganga Contreras, Francisco y Burotto, Juan Felix

básico y medio y modalidades de enseñanza.

- Proponer, asignar y distribuir los recursos que la ley de Presupuestos consulta anualmente para las Instituciones de Educación Superior.

- Mantener un registro Nacional de Instituciones de Educación Superior.

- Diseñar, proponer e implementar programas de fortalecimiento y desarrollo de las Instituciones de Educación Superior, sin prejuicio de la autonomía de estas entidades.

- Establecer las condiciones y desarrollar la normativa que permita la existencia y funcionamiento de los mecanismos que aseguren la calidad de las Instituciones de Educación Superior.

- Cumplir las otras funciones que le encomiende la ley.

b) Contraloría General de la República

Según lo consigna el propio organismo (Contraloría General de la República 2010), esta repartición tiene como misión, dos grandes aspectos:

b.1) Fortalecimiento del Estado de Derecho: pues es un órgano superior de control de la Administración, constitucionalmente autónomo. Desempeña diversas funciones que el ordenamiento jurídico le ha encargado para el fortalecimiento del Estado de Derecho y que están destinadas a hacer respetar el principio de juridicidad; resguardar el patrimonio público; velar por el cumplimiento del principio de probidad administrativa y asegurar la transparencia de la información que generan los órganos públicos.

b.2) Sistema de control: como Entidad Fiscalizadora Superior, constituye un elemento fundamental del sistema nacional de control y en ese sentido, ejerce sus funciones con el fin de promover la certeza, estabilidad y predictibilidad de las decisiones administrativas, y por lo mismo, se convierten en un mecanismo de protección de las garantías fundamentales de los ciudadanos en sus relaciones con la Administración del Estado.

Estos grandes objetivos se logran por medio de los siguientes propósitos estratégicos:

- Fortalecer el principio de probidad administrativa y de la transparencia de la gestión pública.

- Mejorar la capacidad operativa de la Institución.

- Reestructurar el sistema de incentivos al desempeño de los funcionarios.

- Contribuir al proceso de modernización del Estado.

- Ayudar a promover el bien común, al proceso permanente de modernización del Estado.

c) Servicio Nacional del Consumidor (SERNAC)

De acuerdo a la Ley 19496, que "establece normas sobre protección de los derechos de los consumidores", promulgada el 07 de febrero de 1997, el Servicio Nacional del Consumidor será un servicio público funcionalmente descentralizado y desconcentrado territorialmente en todas las regiones del país, con personalidad jurídica y patrimonio propio, sujeto a la supervigilancia del Presidente de la República a través del Ministerio de Economía, Fomento y Reconstrucción. Este organismo público deberá asumir, entre otras, las siguientes funciones: 
- Formular, realizar y fomentar programas de información y educación al consumidor.

- Realizar, a través de laboratorios o entidades especializadas, de reconocida solvencia, análisis selectivos de los productos que se ofrezcan en el mercado en relación a su composición, contenido neto y otras características.

- Recopilar, elaborar, procesar, divulgar y publicar información para facilitar al consumidor un mejor conocimiento de las características de la comercialización de los bienes y servicios que se ofrecen en el mercado.

- Recibir reclamos de consumidores que consideren lesionados sus derechos y dar a conocer al proveedor respectivo el motivo de inconformidad a fin de que voluntariamente pueda concurrir y proponer las alternativas de solución que estime convenientes. Sobre la base de la respuesta del proveedor reclamado, el Servicio Nacional del Consumidor promoverá un entendimiento voluntario entre las partes. El documento en que dicho acuerdo se haga constar tendrá carácter de transacción extrajudicial y extinguirá, una vez cumplidas sus estipulaciones, la acción del reclamante para perseguir la responsabilidad contravencional del proveedor.

- Velar por el cumplimiento de las disposiciones legales y reglamentarias relacionadas con la protección de los derechos de los consumidores y hacerse parte en aquellas causas que comprometan los intereses generales de los consumidores.

\section{c) Inspección del Trabajo}

De acuerdo a lo establecido por Dirección del Trabajo (2010), se trata de un Servicio Público descentralizado con personalidad jurídica y patrimonio propio. Está sometido a la supervigilancia del Presidente de la República a través del Ministerio del Trabajo y Previsión Social y se rige por su Ley Orgánica (D.F.L. N ${ }^{\circ} 2$, del 30 de mayo de 1967).

Además se señala que su misión está orientada a contribuir a modernizar y hacer más equitativas las relaciones laborales, velando por el cumplimiento normativo, promoviendo la capacidad de autorregulación de las partes, sobre la base de la autonomía colectiva y el desarrollo de relaciones de equilibrio entre los actores el mundo del trabajo, empleador y trabajador.

\section{Resultados}

La teoría de agencia parte de la premisa que en la relación contractual entre principal y agente, se producen divergencias de intereses. Para enfrentar esta realidad, se deben asumir o desarrollar "contratos compensatorios" y desplegar "mecanismos alineadores" o "sistemas neutralizadores" de comportamientos oportunistas, de tal forma, que se posibilite que el agente pueda perseguir los intereses del mandante o principal. En general, estos mecanismos conforman el denominado gobierno de las organizaciones, y dependiendo de donde se ejercen estos dispositivos, pueden ser clasificados en internos y externos. La idea de este apartado será por lo tanto, indagar 
Mecanismos de control externo en las universidades de Chile...

Ganga Contreras, Francisco y Burotto, Juan Felix

acerca de dos grandes temas asociados a mecanismos compensatorios: percepción de los sistemas de control, percepción de los sistemas de control externo.

En cuanto a la Percepción general de los sistemas de control esta temática, se buscaba indagar acerca de la opinión del principal y agente, respecto a la evaluación que realizaban de los sistemas de control de las universidades chilenas. En este orden de ideas, al preguntársele a los encuestados "si consideraban que los sistemas de control de las universidades en Chile son ineficientes", más del $60 \%$ promedio está "de acuerdo" y "totalmente de acuerdo" con esta aseveración; del mismo modo, más del $70 \%$ promedio de los MCC opta por esta opinión (porcentaje que en el caso del agente llega al 38\%). En relación a los rectores, la mayoría de ellos (43\%) no se define en una posición, eligiendo por lo tanto, la alternativa "ni de acuerdo", "ni en desacuerdo". Al utilizar la prueba de Kruskall Wallis, $\mathrm{H}=7,040$, $(p=0,030)$, se pudo detectar que existen diferencias estadísticamente significativas, tal como lo muestra el puntaje mediano que asignan (ver Figura 1).

En cuanto a la Percepción de los sistemas de control externo, la idea era averiguar -en términos generales- la evaluación que realizaban los encuestados respecto del sistema de control externo, para ello, los MCC y agentes disponían de una escala de cuatro niveles: "muy bueno", "bueno", "malo" y "muy malo". Las respuestas se concentraron finalmente en sólo dos alternativas: "bueno" y "malo". A diferencia de la evaluación que se realiza de los sistemas de control interno, en este caso, más del $65 \%$ de lo MCC lo califican como "malo"; opinión que es compartida

\section{Figura 1}

\section{Grado de acuerdo de rectores y MCC, respecto de la ineficiencia de los sistemas de control de las universidades de Chile (en porcentajes)}

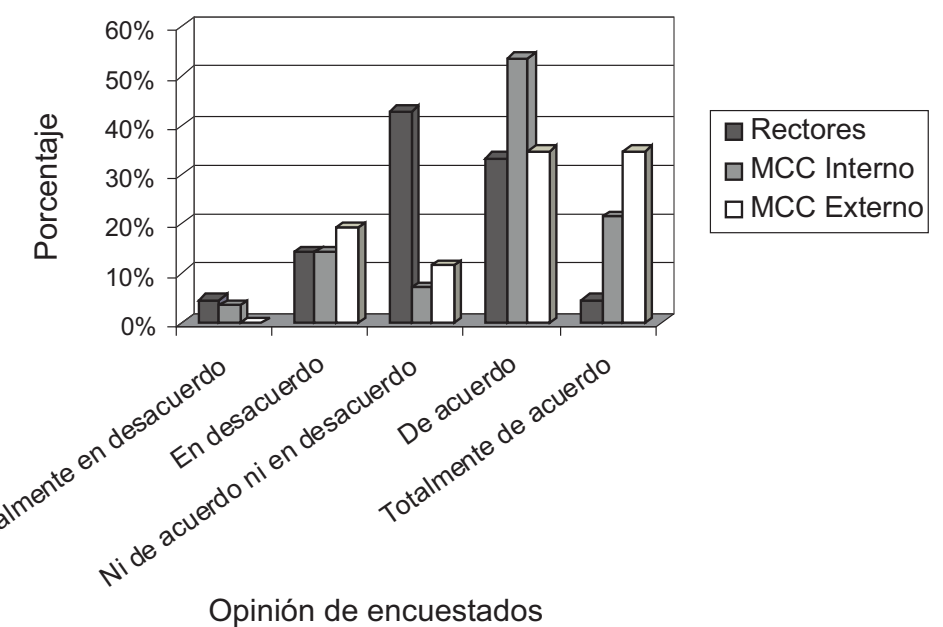

Fuente: Diseño propio, basado en encuesta aplicada a rectores y MCC de las universidades chilenas. 
por los rectores en porcentajes que superan el $52 \%$. Como se puede observar en la Figura 2, la opinión de los participantes sobre esta materia, es independiente del cargo que ocupan, hecho que queda ratificado con la prueba Chi Cuadrado de independencia $\chi 2=1,07(p=0,59)$.

Adicionalmente, se estimó conveniente realizar un parangón para determinar si existen diferencias entre lo que opinan los MCC de las universidades privadas, respecto de las universidades tradicionales, y en este orden de cosas, se pudo constatar que prácticamente el $70 \%$ de los primeros califican como "bueno" el sistema de control externo, apreciación totalmente contraria a la de los MCC de las universidades tradicionales, quienes en más del $80 \%$ lo evalúan como "malo", es decir, los MCC de las instituciones del sector privado, poseen una opinión significativamente más favorable de los controles externos, que los MCC de las instituciones tradicionales. Lo anterior queda ratificado al aplicar la prueba Chi-Cuadrado de independencia, dado que ésta con- firma que existe diferencia entre los MCC, en su opinión sobre la calidad del sistema de control externo, dependiendo del tipo de universidad, situación demostrada además por lo estadígrafos $\chi 2=9,82 \mathrm{y}$ $p=0,0017$ (ver Figura 3).

Las diferencias detectadas en el caso del principal de las universidades tradicionales y privadas, no se producen en la opinión de los rectores, respecto de la valoración que hacen de los sistemas de control externo, y tal como puede observarse en la Figura 4, prácticamente realizan la misma evaluación, con un claro equilibrio entre las alternativas "bueno" y "malo" (sólo se percibe una leve diferencia entre los que opinan los rectores de las universidades tradicionales, que valoran en un $53 \%$ como "malo" los sistemas de control externo).

Complementariamente, se intentó conocer la percepción de los rectores y MCC, respecto del nivel de control que ejercen organismos externos vinculados con la educación superior, tales como: Ministerio de Educación, Contraloría Ge-

Figura 2

Evaluación de los rectores y MCC de las universidades chilenas, del sistema de control externo (en porcentajes)

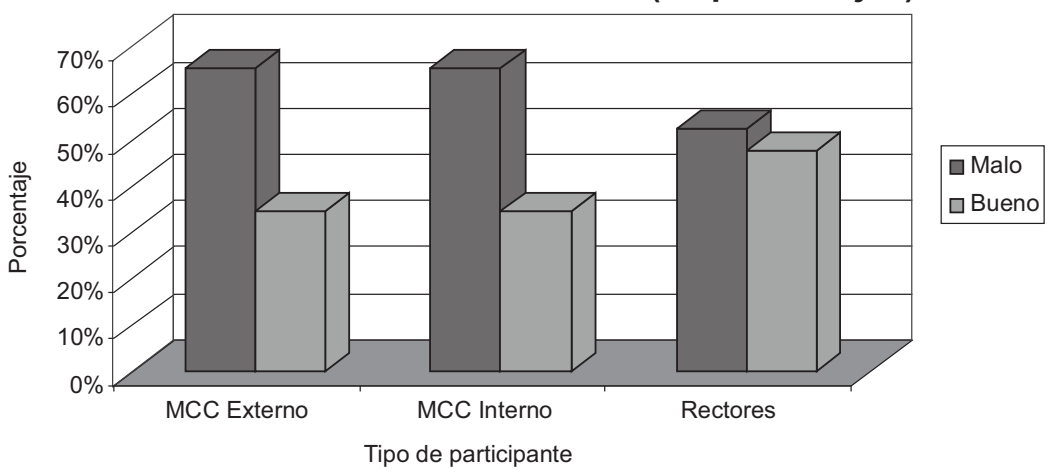

Fuente: Diseño propio, basado en encuesta aplicada a rectores y MCC de las universidades chilenas. 
Mecanismos de control externo en las universidades de Chile...

Ganga Contreras, Francisco y Burotto, Juan Felix

Figura 3

Evaluación de los MCC, por tipo de universidad, del sistema de control externo (en porcentajes)

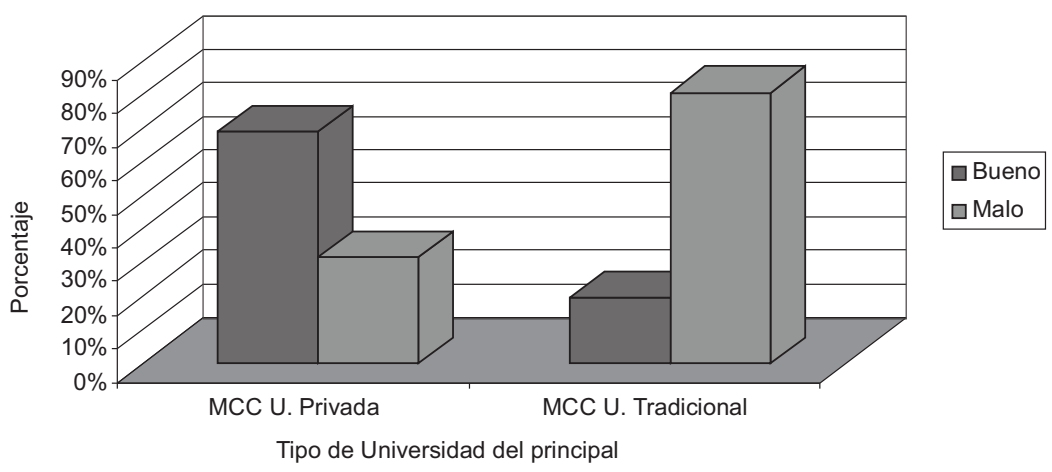

Fuente: Diseño propio, basado en encuesta aplicada a rectores y MCC de las universidades chilenas.

Figura 4

Evaluación de los rectores, por tipo de universidad, del sistema de control externo (en porcentajes)

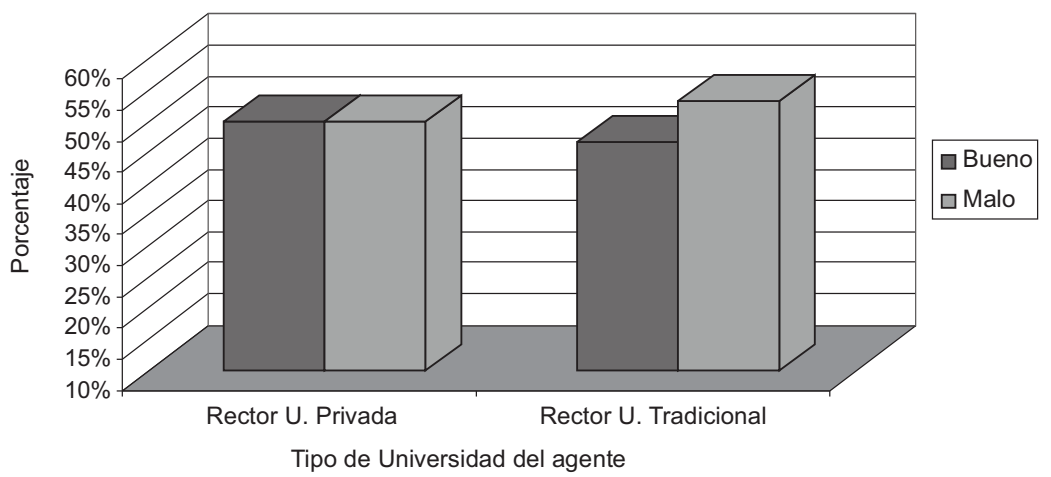

Fuente: Diseño propio, basado en encuesta aplicada a rectores y MCC de las universidades chilenas.

neral de la República, Servicio Nacional de Consumidor y la Inspección del Trabajo. Para ello se les solicitó a los MCC y agentes que determinaran el grado de control que estas instituciones ejercían, en una escala que consideraba cinco niveles: "ningún control", "poco control", "control medio", "suficiente control" y "mucho control". Los resultados permiten aseverar que en términos agregados, en promedio más del $24 \%$ de los encuestados considera que estos organismos ejercen "ningún control" (son los MCC internos los que mayoritariamente optan por esta evaluación, con prácticamente el $30 \%$ ), y en esta misma dirección, un $30,3 \%$ promedio, sostiene que hay "poco control" (mayoritariamente los MCC externos se inclinan por esta alternativa con casi el $40 \%$ ). A su vez, llama la atención 
que en promedio un poco más de $1 / 4$ de los encuestados considera que los organismos externos ejercen "suficiente control" y "mucho control", siendo en este caso los rectores, los que le dan la mayor ponderación (un poco más del $30 \%$ ), mientras los MCC internos y externos comparten cada uno el $27 \%$. En términos estadísticos, se puede adicionar que la prueba Chi Cuadrado entregó el valor $\chi 2=1,94$ $(p=0,93)$, lo cual demuestra que la opinión sobre el nivel de control que ejercen los organismos externos, no depende del cargo, ya sea principal y o agente (ver Figura 5).

Un análisis específico de la percepción que tienen los agentes y principales, del nivel de control que ejercen determinados organismos públicos en las instituciones de educación superior, se presenta a continuación.

Respecto al Grado de control que ejerce el Ministerio de Educación (Mineduc), la prueba Chi Cuadrado con $\chi 2=$ 1,94 y con $(p=0,92)$, corroboran que la opinión de los encuestados es indepen- dientes del cargo ostentando, en cuanto al nivel de control que despliega el Mineduc frente a las universidades de Chile; sin embargo, llama la atención el promedio del $12 \%$ de los encuestados que considera que este organismo ejerce "ningún control" (los más críticos son los MCC internos, quienes en casi el $20 \%$ optan por esta alternativa) y prácticamente el $35 \%$ estima que se ejerce "poco control" (de ellos casi el $40 \%$ corresponden a MCC externos). En todo caso, más de la mitad promedio de los encuestados, considera que existe un grado de "control medio" o "suficiente control", concentrándose esta respuesta primordialmente en los rectores con casi el 60\% (ver Tabla 1).

En relación al grado de control que ejerce la Contraloría General de Republica, sorprende el alto porcentaje de los participantes que considera que este organismo ejerce "ningún control" ( $35 \%$ de los rectores y MCC externos y el $41 \%$ de los MCC internos). Como contrapartida, más de la mitad de los rectores considera que la Contraloría General de la Repúbli-

Figura 5

Grado de control que ejercen organismos externos, vinculados con la educación superior, en las universidades chilenas (en porcentajes)

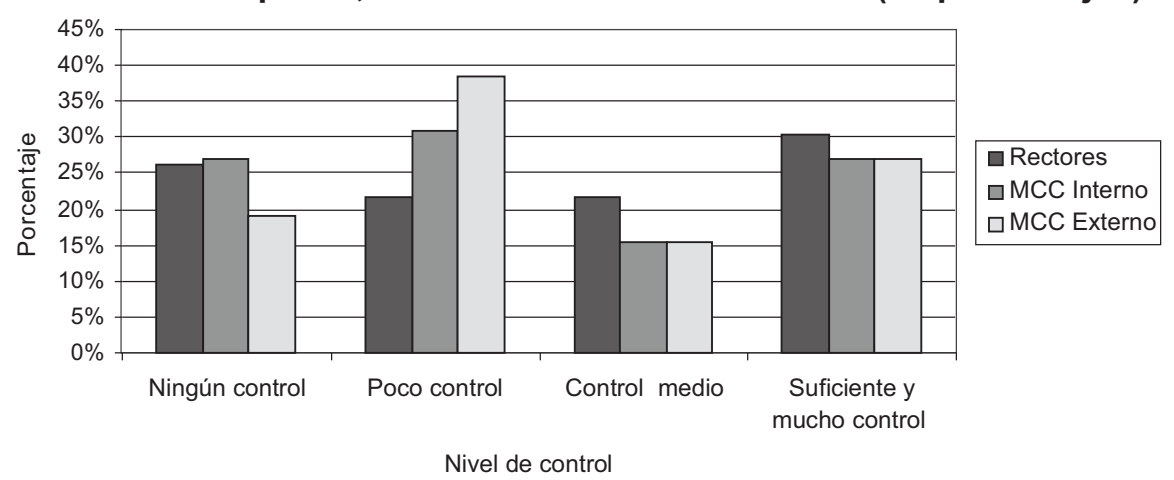

Fuente: Diseño propio, basado en encuesta aplicada a rectores y MCC de las universidades chilenas. 
Mecanismos de control externo en las universidades de Chile...

Ganga Contreras, Francisco y Burotto, Juan Felix

Tabla 1

Grado de control que ejerce el Ministerio de Educación, según rectores y $\mathrm{MCC}$

\begin{tabular}{lccc}
\hline Nivel de control & Rectores & MCC Interno & MCC Externo \\
\hline Ningún control & $9,1 \%$ & $16,7 \%$ & $11,5 \%$ \\
Poco control & $31,8 \%$ & $29,2 \%$ & $38,5 \%$ \\
Control medio & $36,4 \%$ & $25,0 \%$ & $23,1 \%$ \\
Suficiente control & $22,7 \%$ & $29,2 \%$ & $26,9 \%$ \\
\hline
\end{tabular}

Fuente: Diseño propio, basado en encuesta aplicada a rectores y MCC de las universidades chilenas.

ca ejerce "suficiente y mucho control", porcentaje que alcanza al $33 \%$ y $46 \%$ en el caso de los MCC interno y MCC externo, respectivamente. En todo caso, la prueba Chi Cuadrado, con $\chi 2=2,31 \mathrm{y}$ $(p=0,68)$, confirman que la opinión entre rectores y MCC -respecto del nivel de control que ejerce este organismo público sobre las universidades- es independiente al rol que ocupan en sus respectivas casas de estudios (ver Tabla 2).

La percepción de los encuestados, respecto de nivel de control que ejerce el Servicio Nacional del Consumidor (Sernac) no difiere entre el principal y el agente, tal como queda demostrado con los estadígrafos $\chi 2=5,5$ y $p=0,24$. No obstante, se mantienen porcentajes significativos de participantes que estiman que Sernac "no controla nada", con un $20 \%$ promedio de las opiniones (dentro de éstos, el $26 \%$ de los MCC internos y un poco más del $11 \%$ de los MCC externos, participan de esta idea). En esta misma línea de análisis, el mayor porcentaje promedio se aglutina en la alternativa "poco control" con un 52\% promedio, concentrándose el mayor porcentaje, para esta alternativa, en los MCC externos, con casi el $70 \%$ (en el caso de los MCC internos, no llega al 50\%). Los recto- res por su parte, se centran más en las alternativas "poco control" y "medio y suficiente control", con casi un $40 \%$ para cada opción (ver Tabla 3).

En esta última situación referida al el Servicio Nacional del Consumidor (Sernac) se mantiene el alto porcentaje de encuestados que estima que este organismo público no ejercen control en la gestión de las universidades, percepción que tienen prácticamente el $25 \%$ promedio de los rectores y MCC (de éstos, son los agentes los que tienen el mayor porcentaje de opinión centrada en esta alternativa, con un poco más del $30 \%$ ). No obstante, el mayor porcentaje de las respuestas se aglutina entorno a la alternativa "control medio" y "suficiente control", con un promedio del $43 \%$, ejerciendo en este porcentaje, mucha influencia la opinión de los rectores, los cuales casi en un $60 \%$ evalúan con esta opción, contra el $36 \%$ de los MCC. A pesar de estas diferencias observadas, se puede puntualizar que desde el punto de vista de los estadígrafos $\chi 2=6,12$, y $(p=0,186)$, la opinión de los MCC y rectores no es diferente entre ellos (ver Tabla 4).

En la Tabla 5, se presenta una síntesis de la percepción de rectores y MCC, 
Tabla 2

Grado de control que ejerce la Contraloría General de la República, según rectores y $\mathrm{MCC}$

\begin{tabular}{lccc}
\hline Nivel de control & Rectores & MCC Interno & MCC Externo \\
\hline Ningún control & $34,8 \%$ & $40,7 \%$ & $34,6 \%$ \\
Poco o medio control & $13,0 \%$ & $25,9 \%$ & $19,2 \%$ \\
Suficiente o mucho control & $52,2 \%$ & $33,3 \%$ & $46,2 \%$ \\
\hline
\end{tabular}

Fuente: Diseño propio, basado en encuesta aplicada a rectores y MCC de las universidades chilenas.

Tabla 3

Grado de control que ejerce el Servicio Nacional del Consumidor, según rectores y $M C C$

\begin{tabular}{lccc}
\hline Nivel de control & Rectores & MCC Interno & MCC Externo \\
\hline Ningún control & $21,7 \%$ & $25,9 \%$ & $11,5 \%$ \\
Poco control & $39,1 \%$ & $48,1 \%$ & $69,2 \%$ \\
Suficiente o medio control & $39,1 \%$ & $25,9 \%$ & $19,2 \%$ \\
\hline
\end{tabular}

Fuente: Diseño propio, basado en encuesta aplicada a rectores y MCC de las universidades chilenas.

Tabla 4

Grado de control que ejerce la Inspección del Trabajo, según rectores y $\mathrm{MCC}$

\begin{tabular}{lccc}
\hline Nivel de control & Rectores & MCC Interno & MCC Externo \\
\hline Ningún control & $30,4 \%$ & $24,0 \%$ & $20,0 \%$ \\
Poco control & $13,0 \%$ & $40,0 \%$ & $44,0 \%$ \\
Suficiente o medio control & $56,5 \%$ & $36,0 \%$ & $36,0 \%$ \\
\hline
\end{tabular}

Fuente: Diseño propio, basado en encuesta aplicada a rectores y MCC de las universidades chilenas.

en relación a los sistemas de control ( $\mathrm{y}$ algunos organismos públicos dedicado a ello), analizados previamente.

Al visualizar la diversidad de información acopiada y presentada, en cuanto a los dispositivos de control del sistema universitario, en este caso y de modo muy general, se pudo constatar que los MCC valoran más negativamente los sistemas de control, que los rectores de las universidades chilenas, en todo caso, desde el punto de vista estadístico, en cinco de las seis variables analizadas, no se logró de- tectar diferencias estadísticamente significativas.

\section{Conclusiones}

La investigación realizada muestra resultados que parecen coincidir con los presupuestos éticos que se comprometían en la introducción, a saber, la adhesión a lo verdadero, como era esperable en individuos en los cargos de Principal y Agente en los gobiernos corporativos universitarios, pero además una interesante 
Mecanismos de control externo en las universidades de Chile...

Ganga Contreras, Francisco y Burotto, Juan Felix

Tabla 5

Percepción de agente y principal de los sistemas de control

\begin{tabular}{|c|c|c|c|c|c|c|}
\hline \multirow[t]{2}{*}{ Tópico evaluado } & \multicolumn{2}{|c|}{$\begin{array}{l}\text { Prueba de } \\
\text { Kruskall Wallis }\end{array}$} & \multicolumn{2}{|c|}{$\begin{array}{l}\text { Prueba de } \\
\text { Chi Cuadrado }\end{array}$} & \multicolumn{2}{|c|}{$\begin{array}{c}\text { Dif. } \\
\text { Estadísticamente } \\
\text { significativas }\end{array}$} \\
\hline & $\mathbf{H}$ & p & $\times 2$ & p & Sí & No \\
\hline a) Percepción de los sistemas de control. & 7,040 & 0,030 & & & $x$ & \\
\hline $\begin{array}{l}\text { b) Percepción de los sistemas de control } \\
\text { externo. }\end{array}$ & & & 1,070 & 0,590 & & $x$ \\
\hline $\begin{array}{l}\text { c) Grado de control que ejerce } \\
\text { el Ministerio de Educación (Mineduc). }\end{array}$ & & & 1,940 & 0,920 & & $x$ \\
\hline $\begin{array}{l}\text { d) Grado de control que ejerce } \\
\text { la Contraloría General de la República. }\end{array}$ & & & 2,31 & 0,680 & & $x$ \\
\hline $\begin{array}{l}\text { e) Grado de control que ejerce el Servicio } \\
\text { Nacional del Consumidor (Sernac). }\end{array}$ & & & 5,50 & 0,240 & & $\mathrm{X}$ \\
\hline $\begin{array}{l}\text { f) Grado de control que ejerce } \\
\text { la Inspección del Trabajo. }\end{array}$ & & & 6,12 & 0,182 & & $\mathrm{X}$ \\
\hline
\end{tabular}

Fuente: Diseño propio, basado en encuesta aplicada a rectores y MCC de las universidades chilenas.

similitud en las percepciones en ambos roles y en donde, por añadidura, se enangosta la gravitación del carácter público o privado, en que puede seccionarse el universo investigado.

De lo anterior se colige de manera elocuente que tanto los MCC y los Rectores no otorgan demasiada importancia a los mecanismos de control externo. Se advierte, a las claras, que salvo los mecanismos de certificación de calidad y, en correspondencia con éstos, la concursabilidad para fondos públicos, los ejecutivos universitarios no encuentran que tales mecanismos externos sean per se dispositivos que superen la coyuntura que permea el tipo de Estado que actualmente exhibe Chile, en donde el Ministerio de Educación, tiene un papel de fiscalización morigerado como mecanismo controlador, ya que actúa en la acreditación que dispensa a terceros: los organismos acreditadores o certificadores de calidad de lo universitario.

Para una mayor comprensión de los resultados y a modo de desenlace, se puede indicar que ha quedado nítidamente de manifiesto que los actores creen que en general los sistemas de control que tienen las universidades son ineficientes, posición que asume más del $70 \%$ promedio de los MCC y un porcentaje no menor, que alcanza el $43 \%$ de los rectores. Esta opinión negativa de los sistemas de control, se extrapola a la apreciación que se tiene de los sistemas de control externo, pues, más del $65 \%$ del principal lo evalúan como "malo" y en el caso del agente, el porcentaje sobrepasa el $50 \%$. No obstante, se debe reconocer 
que esta situación es algo diferente cuando nos focalizamos en los MCC de las universidades privadas, debido a que su opinión es mayoritariamente positiva. También es interesante constatar que los organismos públicos que deben ejercer algún nivel de control en las universidades (Ministerio de Educación, Contraloría General de la República, Servicio Nacional del Consumidor e Inspección del Trabajo), resultan muy mal evaluados, pues en general se considera que ellos ejercen "poco o ningún control".

Sin entrar a juicios de valor, que escapan a los propósitos de esta indagación nuestra, se hace necesario destacar que en materia de educación superior el país parece adherir a una concepción que se ampara en los paradigmas del libre mercado en lo educacional. Si es así -y ello ameritaría un escrutinio científico al respectola menor incidencia subjetiva del control externo sería explicable. De otro modo, también se hace evidente que los mecanismos alineadores internos deben tomar mayor fuerza para compensar la escasa gravitación otorgada a los externos: la organización sabe de lo imprescindible de esa alineación de Principal y Agente y un estudio futuro debería dar cuenta de si este aserto es, como conjeturamos, sostenible. En todo caso, el justiprecio de este trabajo alcanzaría su mayor relieve si, desde él, no sólo se contrasta una teoría sino que se visualiza en la práctica su utilidad y, todavía más, su amplia y polifacética potencialidad noológica en el complejo espacio de las conductas corporativas, esto es, la gestión del conocimiento gerencial y su traducción en la órbita de los gobiernos universitarios.

\section{Referencias bibliográficas}

Albrecht, Douglas y Ziderman, Adrian (1992). Financing universities in developing countries. The World Bank. PHREE Background Paper Series.

Azofra, Valentín., y Santamaría, Marcos (2004). "El gobierno de las cajas de ahorro españolas". Universia Business Review. № 2 .

Crespí, Rafel y García-Cestona, Miguel (2002). "Propiedad y control: una perspectiva europea". Revista Vasca de Economía Ekonomiaz, $N^{\circ} 50.2^{\circ}$ Cuatrimestral.

Contraloría General de la República (__ $)$. Información institucional. Disponible en Internet: http://www.contraloria.cl/NewPortal2/portal2/appmanager/portalCGR/portal? nfpb=true\& pageLabel= MIniciov1 (Accesado en: Enero, 2009).

Cuervo, Alvaro; Fernández, Ana y Gómez, Silvia (2002). "Mecanismos externos de control de la empresa: el papel de los bancos y el mercado de control en entorno de baja protección del inversor". Revista Vasca de Economía Ekonomiaz, $N^{\circ} 50.2^{\circ}$ Cuatrimestre.

Demsetz, Harold (1983). "The structure of ownership and theory of the firm". Journal of Law and Economics. $\mathrm{N}^{\circ}$ 26.

Fama, Eugene y Jensen, Michael (1983). "Separation of ownership and control". Journal of Law and Economics. Vol. XXVI.

Fama, Eugene (1980). "Agency problems and the theory of the firm". Journal of Political Economy, $\mathrm{N}^{\circ} 88$.

Fernández, Isabel y Gómez-Anson, S. (1998). "El gobierno corporativo: la supervisión y control de las actuaciones gerenciales". Revista Economía Aragonesa, $N^{\circ} 5$. 
Mecanismos de control externo en las universidades de Chile...

Ganga Contreras, Francisco y Burotto, Juan Felix

Fernández, Enrique; Suárez, Eugenia y Ventura, José (2001). "Discrecionalidad directiva en las mutuas de accidentes del trabajo". Cuadernos de Economía y Dirección de Empresas. № 10.

Fogelberg, Graeme (1980). "Ownership and control in 43 of New Zealand's largest companies". New Zeeland Journal of Business. 2.

Forbes, Daniel y Milliken, Frances (1999). "Cognition and corporate governance: understanding boards of director as strategic decision -making groups". Academy of Management Review. $\mathrm{N}^{\circ} 24$.

Giménez, Isabel (2003). "El gobierno corporativo, el control de las empresas y la defensa de los derechos de los accionistas minoritarios". Revista Valenciana de Economía y Hacienda. $\mathrm{N}^{\circ}$ 9-III. España.

Hart, Oliver (1983). “The market mechanism as an incentive scheme". Bell Journal of Economics. N ${ }^{\circ} 14$.

Hoskisson, Robert y Turk, Tomaz (1990). "Corporate restructuring: governance and control limits of internal market". Academy of Management Review. 15.

Inspección del Trabajo de Chile (_ $)$. Información institucional. Disponible en Internet:

http://www.dt.gob.cl/1601/w3-propertyname-2299.html (Accesado en: Enero, 2009).

Jensen, Michael y Williams, Meckling (1976). Teoría de la empresa: La gerencia; costes de agencia y estructura de la propiedad. Apunte proporcionado en la Asignatura Economía Financiera del Programa de Doctorado en Nuevas Tendencias en Dirección de Empresas. Universidad de Valladolid. España.

Jensen, Michael (1993). "The modern industrial revolution, exit, and the Failure of Internal Control Systems". The Journal of Finance. $\mathrm{N}^{\circ} 48$.
Lozano, María (2005). "El mercado de control empresarial ante el conflicto de agencia accionista-directivo". Tribuna de Economía. ICE. N ${ }^{\circ} 823$.

Maroto, Juan y Melle, Mónica (2001). "Sistemas financieros y economía real: modelos de relación y gobierno de las empresas". Revista Vasca de Economía Ekonomiaz. $\mathrm{N}^{\circ}$ 50. $2^{\circ}$ Cuatrimestre.

Maroto, Juan; Melle, Mónica; Moreno, Ignacio; Rodríguez, José (2006). "Grado de competencia, presión de la deuda y productividad empresarial: un análisis empírico desde la perspectiva del gobierno corporativo". Panorama Socioeconómico. Año 24. №33.

Méndez, Carlos (1988). Metodología. Guía para elaborar diseños de investigación en ciencias económicas, contables y administrativas. Colombia. Editorial McGraw-Hill, Bogotá.

Ministerio de Educación de Chile (__ $)$. Estadísticas institucionales. Disponible en Internet: http://www.mineduc.cl/ (Accesado en: Octubre, 2003).

Ministerio de Educación de Chile (__ _ $)$. Estadísticas institucionales. Disponible en Internet: http://www.mineduc.cl/ (Accesado en: Mayo, 2006).

Ministerio de Educación de Chile. División de Educación Superior (__ $)$. Estadísticas institucionales. Disponible en Internet: http://www.mineduc.cl/ (Accesado en: Mayo, 2006).

Ministerio de Educación de Chile. División de Educación Superior (_ torio de Instituciones. Disponible en Internet: http://www.divesup.cl/index.php?option=com_content\&view= article\&id=79\&ltemid=131 (Accesado en: Mayo, 2009).

Ministerio de Educación de Chile Marco legal. Disponible en Internet: http://w3app.mineduc.cl/edusup/instituciones/inst_marc_lega.html (Accesado en: Enero, 2005). 
Ministerio de Educación de Chile ( D.F.L. $N^{\circ}$ 1. Fija normas sobre universidades. Disponible en Internet: http://www.divesup.cl/images/archivos/leyes/6.\%20dfl\%201_1981.pdf (Accesado en: Enero, 2008).

Ministerio de Educación de Chile ( D.F.L. $\mathrm{N}^{\circ} 4$. Fija normas sobre financiamiento de las universidades. Disponible en Internet: http://www.educacionsuperiorchile.cl/docs/doc2004/ DFL_4.doc (Accesado en: Enero, 2008).

Ministerio de Educación de Chile (__ $)$. Información institucional. Disponible en Internet: http://www.mineduc.cl/index 0.php?id_portal=1 (Accesado en: Enero, 2009)

Montoro, Juan y García, Francisco (2001). "EI papel del sector no lucrativo en la provisión voluntaria de bienes públicos". Revista Valenciana de Economía y Hacienda (RVEH). $\mathrm{N}^{\circ} 2$.

Monks, Robert y Minow, Nell (1995). Corporate governance. Cambridhe, MA: Blackwell Business.

Morin, Edgar (2008). La Méthode. Paris, Seuil.

Ocaña, Carlos y Salas, Vicente (1983). "La teoría de la agencia. Aplicación a las empresas públicas españolas". Cuadernos Económicos del ICE. Vol. 22-23.

Ortega-Argilés, Raquel; Moreno, Rosina y Surinach, Jordi (2006). "La aplicación de los mecanismos de control de los problemas de agencia. Un análisis de las empresas manufactureras españo- las". Tribuna de Economía. ICE. N ${ }^{\circ}$ 829.

Palacín, María (2004). El gobierno de empresa: mecanismos de control interno y mecanismos de control externo. Esic Market. $N^{\circ} 113$. Disponible en internet: http://www.personal.us.es/marpalsan/investigacion.htm (Accesado en: Junio 2005).

Servicio Nacional del Consumidor ( Información institucional. Disponible en Internet: http://www.sernac.cl/ acercade/index.php (Accesado en: Enero, 2009).

Schellenger, Michael H.; David D. Wood y Ahmad Tashakori (1989). "Board of director composition, shareholders weath, and dividend policy". Journal of Management, Vol. 15.

Shivdasani, Anil (1993). "Board composition ownership structure and hostile takeovers". Journal of Accounting and Economics. $N^{\circ} 16$.

Shleifer, Andrei y Vishny, Robert (1997). "A survey of corporate governance". The Journal of Finance. $\mathrm{N}^{\circ} 52$.

Shleifer, Andrei y Vishny, Robert (1995). "A survey of corporate governance". Harvard Institute of Economic Research. Discussion Paper N ${ }^{\circ} 1741$.

Zahra, Shaker y Pearce, John (1989). "Board of directors and corporate financial performance: a review and integrate mode". Journal of Management. Vol. 15. $\mathrm{N}^{\circ} 2$. 